\title{
Flora de comunidades de macroalgas lóticas de fragmentos florestais remanescentes da região noroeste do Estado de São Paulo, Brasil
}

\author{
Fernanda Vital Ramos de Almeida ${ }^{1}$, Orlando Necchi Jr. ${ }^{1,2}$ e Luis Henrique Zanini Branco ${ }^{1}$
}

Recebido: 18.11.2010; aceito: 10.11.2011

\begin{abstract}
Lotic macroalgal communities flora from remnant forest fragments from the northwest region of São Paulo State, Brazil). Seventeen streams in 12 forest fragments were sampled during the dry season (June to August of 2007 and of 2008). Sixteen species belonging to 14 genera were found. Cyanophyta and Chlorophyta were the best represented algal division (44 and 38\%, respectively), followed by Rhodophyta (12\%) and Heterokontophyta (6\%). Global species number was low, probably due to predominance of sand-clay substrates $(65 \%)$ and of shaded stream segments $(53 \%)$. Most species $(69 \%)$ occurred in only one stream, a pattern often observed in lotic macroalgal communities. Vaucheria pseudogeminata was reported for the first time for Brazil, whereas Trichocoleus sociatus for this region. Comparing these macroalgal flora with those from other biomes of São Paulo State, the highest similarity was with Tropical Forest (56\% in common), as expected because it is the nearest biome and also constituted by Semideciduous Seasonal Forest.
\end{abstract}

Key words: Diversity, macroalgae, Semidecidual Seasonal Forest, stream

RESUMO - (Flora de comunidades de macroalgas lóticas de fragmentos florestais da região noroeste do Estado de São Paulo, Brasil). Foram amostrados 17 riachos, em 12 fragmentos durante a estação seca (junho a agosto de 2007 e de 2008). Foram identificadas 16 espécies de macroalgas, pertencentes a 14 gêneros. Cyanophyta e Chlorophyta foram os grupos mais representativos (44 e 38\%, respectivamente), seguidos por Rhodophyta (12\%) e Heterokontophyta (6\%). O número global de espécies foi baixo, fato atribuído à predominância de substrato areno-argiloso (65\%) e de trechos sombreados (53\%). A maioria das espécies (69\%) ocorreu exclusivamente em um único riacho, padrão frequentemente observado para macroalgas lóticas. Vaucheria pseudogeminata foi reportada pela primeira vez para o Brasil e Trichocoleus sociatus para a região noroeste do Estado de São Paulo. Comparando a flora de macroalgas encontrada com aquelas de outras regiões/ biomas do Estado de São Paulo, notou-se maior semelhança com Floresta Tropical (56\% espécies em comum), conforme esperado, por ser o bioma mais próximo e composto também por Floresta Estacional Semidecidual.

Palavras-chave: Diversidade, Floresta Estacional Semidecidual, macroalga, riacho

\section{Introdução}

Um dos componentes importantes dos ambientes lóticos são as algas, e embora existam dificuldades de amostragem (Round 1965), são crescentes os estudos realizados com macroalgas nesses ambientes, inclusive no Brasil (Branco \& Necchi Jr. 1996, Necchi Jr. et al. 2003, Krupek et al. 2007, Necchi Jr. et al. 2008, Peres et al. 2008).

Muitos desses trabalhos envolvem a região noroeste do Estado de São Paulo, sendo que estudos florísticos e ecológicos sobre comunidades de macroalgas lóticas foram feitos em um riacho (Necchi Jr. et al. 1991), uma bacia de drenagem (Necchi Jr. 1993, Necchi Jr. et al. 1994b), em três bacias de drenagens (Branco \& Necchi Jr. 1998) e em toda a região noroeste do Estado de São Paulo (Necchi Jr. et al. 1995, 1997). Trabalhos envolvendo diferentes biomas ou regiões do Estado de São Paulo, incluindo a região noroeste, foram desenvolvidos para as macroalgas em geral, abordando aspectos taxonômicos e ecológicos (Necchi Jr. et al. 2000) assim como para grupos específicos, entre eles Cyanophyta (Branco et al. 1999, 2001), Rhodophyta (Necchi Jr. et al. 1999), Characeae (Chlorophyta) (Vieira et al. 2002, 2003), Chaetophoraceae (Chlorophyta) (Branco et al. 2002) e

1. Universidade Estadual Paulista “Júlio de Mesquita Filho", Instituto de Biociências, Letras e Ciências Exatas, Departamento de Zoologia e Botânica, Rua Cristóvão Colombo 2265, 15054-000 São José do Rio Preto, SP, Brasil

2. Autor para correspondência: orlando@ibilce.unesp.br 
os gêneros Vaucheria (Heterokontophyta) (Necchi Jr. et al. 2001) e Microspora (Chlorophyta) (Necchi Jr. et al. 2002). Embora esse conjunto de trabalhos envolva comunidades de macroalgas da região noroeste, ainda não foi realizada qualquer abordagem sobre aspectos florísticos de macroalgas lóticas em fragmentos florestais remanescentes da região.

O noroeste de São Paulo é, portanto, um bom exemplo de região que sofre com a fragmentação de hábitats. Essa região possui apenas $9 \%$ de sua área original, sendo o restante substituído por pastagem, monoculturas e área urbana (Probio 1998). Portanto, é essencial a realização de estudos para o conhecimento dessas áreas, para que se evite a perda de biodiversidade e possibilite o gerenciamento do uso sustentável desses fragmentos. Os remanescentes da região noroeste são constituídos especialmente por Floresta Estacional Semidecidual, um tipo de floresta tropical seca, caracterizada pela ausência de coníferas e pela perda parcial das folhas, em decorrência da baixa precipitação pluviométrica no inverno. Além disso, segundo Rambadi \& Oliveira (2003), ela é importante pela singularidade de espécies, pelo nível de destruição e pela importância econômica.

O presente estudo teve como objetivo realizar o levantamento florístico das comunidades de macroalgas lóticas de fragmentos remanescentes florestais da região noroeste do Estado de São Paulo. Entre os biomas/regiões ocorrentes no Estado de São Paulo, amostrados por Necchi Jr. et al. (2000), espera-se que a região estudada apresente maior similaridade florística com Floresta Tropical, que é o bioma que se encontra mais próximo da região amostrada, além de ser composto também por Floresta Estacional Semidecidual. Este trabalho fez parte do projeto temático "Fauna e flora de fragmentos florestais remanescentes do noroeste paulista: base para estudos de conservação da biodiversidade" (Programa BIOTA/FAPESP, Proc. 2004/04820-3).

\section{Material e métodos}

Os limites da região noroeste do Estado de São Paulo (2000'13"'-21'37'14"'S e 48³2'26"$50^{\circ} 26^{\prime} 02^{\prime \prime} \mathrm{W}$ ), incluem integralmente as Unidades de Gerenciamento dos Recursos Hídricos (UGRHIs) do Turvo-Grande e São José dos Dourados e partes das UGHRIs do Baixo Pardo, Baixo Tietê e Tietê-Batalha. Esta área compreende as regiões administrativas dos municípios-sede de São José do Rio Preto, Araçatuba, e parte das regiões administrativas de Bauru (porção norte) e Ribeirão Preto (porção oeste).

O esquema amostral do presente estudo foi constituído por nove fragmentos grandes (designados de G1 a G9, com áreas maiores que 200 ha) e nove pequenos (designados de $\mathrm{P} 1$ a $\mathrm{P} 9$, com áreas entre 50 e 150 ha) (tabela 1, figura 1), divididos em dois blocos de nove fragmentos (que continham tanto fragmentos grandes quanto pequenos), para serem amostrados durante um ano, cada bloco em anos diferentes. Dos 18 fragmentos selecionados, 12 foram efetivamente amostrados, pois em um deles (P3), o riacho estava seco, e cinco outros (P5, P8, G2, G4 e G7) não continham riachos amostráveis (ambientes lênticos, pantanosos ou muito pequenos). Por outro lado, os fragmentos P7, G1, G5, G8 e G9 continham dois riachos amostráveis cada (R1 e R2), totalizando 17 riachos amostrados. Dos 18 fragmentos, dois (um grande - G9 e um pequeno - P4) foram amostrados nos dois anos, de modo a permitir avaliar a representatividade e repetibilidade de cada amostragem. Esses dois fragmentos (denominados fragmentos de referência) foram escolhidos com base no tamanho (G9 é o maior fragmento da região) e por estarem entre aqueles em melhor estado de preservação. Sendo assim, no ano de 2007 foram amostrados os riachos P1, P2, P4, G1R1, G1R2, G3, G5R1, G5R2, G9R1, G9R2, e no ano de 2008 foram amostrados P4, P6, P7R1, P7R2, P9, G6, G8, G9R1 e G9R2. Informações sobre os fragmentos foram obtidos a partir de dados pertencentes ao projeto temático (tabela 1).

Com o intuito de descrever as características do ambiente de amostragem, em cada ponto foram medidas as seguintes variáveis ambientais, conforme procedimentos gerais descritos por Necchi Jr. et al. (2000): latitude e longitude; profundidade e velocidade da correnteza; temperatura da água, turbidez, condutividade, $\mathrm{pH}$ e oxigênio dissolvido. Para cada variável, em cada riacho, foram realizadas três medições, de modo a obter-se a média. Em P7R2 não foi possível realizar as medições devido à grande profundidade do riacho. Dados de sombreamento e substrato também foram tomados para cada ponto de amostragem. O substrato foi estimado em termos percentuais por análise visual do leito do riacho e denominado de acordo com as classes de tamanho de partículas (Gordon et al. 1992). Os dados de sombreamento foram definidos por meio da classificação em uma de quatro classes de 
Tabela 1. Descrição dos pontos de amostragem nos fragmentos remanescentes florestais da região noroeste do Estado de São Paulo, Brasil, amostrados no período de julho a agosto de 2007 e de 2008.

Table 1. Description of the sampling sites in the remnant forest fragments from the northwest region of São Paulo State, Brazil, sampled during the period of July and August of 2007 and of 2008.

\begin{tabular}{|c|c|c|c|c|c|c|c|c|}
\hline $\mathrm{N}^{\mathrm{o}}$ & Localização & $\begin{array}{l}\text { Posição do } \\
\text { riacho no } \\
\text { fragmento }\end{array}$ & $\begin{array}{l}\text { Perímetro } \\
\quad(\mathrm{m})\end{array}$ & $\begin{array}{l}\text { Área } \\
\text { (ha) }\end{array}$ & $\begin{array}{l}\text { Índice de } \\
\text { forma }^{b}\end{array}$ & $\begin{array}{l}\text { Matriz } \\
\text { predominante }\end{array}$ & $\begin{array}{l}\text { Ordem de } \\
\text { Grandeza } \\
1: 50.000^{\circ}\end{array}$ & UGRHI $^{\mathrm{d}}$ \\
\hline P1 & $\begin{array}{l}\text { Santo Antonio do } \\
\text { Aracanguá,Vicentópolis, } \\
\text { Faz. Rancho Alegre, } \\
20^{\circ} 55^{\prime} 22.3^{\prime \prime S} \text { e } 50^{\circ} 20^{\prime} 33.3^{\prime \prime} \mathrm{W}\end{array}$ & borda & $6.098,0$ & 128,2 & 1,52 & pastagem & 1 & Baixo-Tietê \\
\hline P2 & $\begin{array}{l}\text { Macaubal, Ida Iolanda, } \\
\text { Faz. Pauã, } \\
20^{\circ} 44^{\prime} 39.0^{\prime \prime S} \text { e } 49^{\circ} 56^{\prime} 03.5^{\prime \prime} \mathrm{W}\end{array}$ & interior & $4.337,9$ & 66,8 & 1,50 & cana & 1 & Baixo-Tietê \\
\hline $\mathrm{P} 3{ }^{\mathrm{a}}$ & $\begin{array}{l}\text { Votuporanga, Faz.Primavera, } \\
20^{\circ} 31^{\prime} 04.1^{\prime \prime} \text { S e } 50^{\circ} 05^{\prime} 02.5^{\prime} \mathrm{W}\end{array}$ & borda & $6.914,4$ & 112,6 & 1,84 & cana & 1 & Turvo-grande \\
\hline P4 & $\begin{array}{l}\text { Turmalina, Faz. S. João, } \\
20^{\circ} 00^{\prime} 33.6^{\prime} \text { 'S e } 50^{\circ} 25^{\prime} 36.8^{\prime} \mathrm{W}\end{array}$ & borda & $5.111,0$ & 107,9 & 1,39 & pastagem & 4 & Turvo-grande \\
\hline P6 & $\begin{array}{l}\text { Palestina } \\
20^{\circ} 19^{\prime} 17^{\prime \prime} \text { S e } 49^{\circ} 30^{\prime} 50^{\prime \prime} \mathrm{W}\end{array}$ & borda & $4.587,2$ & 95,5 & 1,34 & cana & 1 & Turvo-Grande \\
\hline P7-R1 & $\begin{array}{l}\text { Barretos } \\
20^{\circ} 38^{\prime} 34^{\prime \prime} \text { 'S e } 48^{\circ} 45^{\prime} 22^{\prime \prime} \mathrm{W}\end{array}$ & borda & $4,166,7$ & 90,7 & 1,43 & cana & 1 & Baixo-Pardo \\
\hline P7-R2 & $\begin{array}{l}\text { Barretos } \\
20^{\circ} 38^{\prime} 36^{\prime \prime} \text { S e } 48^{\circ} 45^{\prime} 24^{\prime \prime} \mathrm{W}\end{array}$ & borda & $4,166,7$ & 90,7 & 1,43 & cana & 1 & Baixo-Pardo \\
\hline P9 & $\begin{array}{l}\text { Pindorama } \\
21^{\circ} 13^{\prime} 20^{\prime \prime} \text { S e } 48^{\circ} 55^{\prime} 07^{\prime \prime} \mathrm{W}\end{array}$ & borda & $6.550,6$ & 108,8 & 2,10 & pastagem & 1 & Turvo-Grande \\
\hline G1-R1 & $\begin{array}{l}\text { Novo Horizonte, } \\
\text { Faz. Serrinha } \\
21^{\circ} 31^{\prime} 26^{\prime} \text { 'S e } 49^{\circ} 18^{\prime} 53^{\prime \prime} \mathrm{W}\end{array}$ & borda & $11.866,6$ & 630,7 & 1,34 & cana & 2 & Tietê-Batalha \\
\hline G1-R2 & $\begin{array}{l}\text { Novo Horizonte, } \\
\text { Faz. Serrinha } \\
21^{\circ} 31^{\prime} 26^{\prime} \text { 'S e } 49^{\circ} 18^{\prime} 53^{\prime \prime} \mathrm{W}\end{array}$ & borda & $11.866,6$ & 630,7 & 1,34 & cana & 1 & Tietê-batalha \\
\hline G3 & $\begin{array}{l}\text { Planalto, Faz. Taperão } \\
20^{\circ} 59^{\prime} 26^{\prime} \text { 'S e } 49^{\circ} 58^{\prime} 50^{\prime \prime} \mathrm{W}\end{array}$ & borda & $6.372,4$ & 215,7 & 1,22 & pastagem & 1 & $\begin{array}{r}\text { Baixo-Tietê } \\
\text { continua }\end{array}$ \\
\hline
\end{tabular}


Tabela 1 (continuação)

\begin{tabular}{|c|c|c|c|c|c|c|c|c|}
\hline $\mathrm{N}^{\circ}$ & Localização & $\begin{array}{l}\text { Posição do } \\
\text { riacho no } \\
\text { fragmento }\end{array}$ & $\begin{array}{l}\text { Perímetro } \\
\text { (m) }\end{array}$ & $\begin{array}{l}\text { Área } \\
\text { (ha) }\end{array}$ & $\begin{array}{l}\text { Índice } d \\
\text { forma }^{b}\end{array}$ & $\begin{array}{l}\text { Matriz } \\
\text { predominante }\end{array}$ & $\begin{array}{l}\text { Ordem de } \\
\text { Grandeza } \\
1: 50.000^{\circ}\end{array}$ & $\mathrm{UGRHI}^{\mathrm{d}}$ \\
\hline G5-R1 & $\begin{array}{l}\text { São João de Iracema, } \\
\text { Faz. São Francisco } \\
20^{\circ} 30^{\prime} 30^{\prime} \text { S e } 50^{\circ} 14^{\prime} 00^{\prime \prime} \mathrm{W}\end{array}$ & interior & $41.367,0$ & $1.656,2$ & 2,87 & cana & 2 & $\begin{array}{l}\text { São José do } \\
\text { Dourados }\end{array}$ \\
\hline G5-R2 & $\begin{array}{l}\text { São João de Iracema, } \\
\text { Faz. São Francisco } \\
20^{\circ} 30^{\prime} 37^{\prime} \text { S e } 50^{\circ} 13^{\prime} 44^{\prime \prime} \mathrm{W}\end{array}$ & interior & $41.367,0$ & $1.656,2$ & 2,87 & cana & 3 & $\begin{array}{l}\text { São José do } \\
\text { Dourados }\end{array}$ \\
\hline G6 & $\begin{array}{l}\text { Nova Granada, Faz.São João } \\
20^{\circ} 33^{\prime} 05^{\prime} \text { 'S e } 49^{\circ} 14^{\prime} 39^{\prime \prime} \mathrm{W}\end{array}$ & interior & $43.180,6$ & $1.359,7$ & 5,39 & laranja & 3 & Turvo-Gande \\
\hline G8-R1 & $\begin{array}{l}\text { Bebedouro, Faz. Córrego dos } \\
\text { Bois } \\
20^{\circ} 53^{\prime} 24^{\prime \prime} \text { S e } 48^{\circ} 31^{\prime} 43^{\prime} \text { W }\end{array}$ & interior & $13.755,5$ & 393,9 & 1,97 & cana & 2 & Baixo-Pardo \\
\hline G8-R2 & $\begin{array}{l}\text { Bebedouro, Faz. Córrego dos } \\
\text { Bois } \\
20^{\circ} 53^{\prime} 34^{\prime} \text { 'S e } 48^{\circ} 31^{\prime} 58^{\prime \prime}\end{array}$ & borda & $13.755,5$ & 393,9 & 1,97 & cana & 1 & Baixo-Pardo \\
\hline G9-R1 & $\begin{array}{l}\text { Matão, Faz. Cambuhy } \\
21^{\circ} 36^{\prime} 35^{\prime} \text { 'S e } 48^{\circ} 33^{\prime} 05^{\prime \prime} \mathrm{W}\end{array}$ & borda & $39.128,9$ & $2.189,6$ & 2,36 & laranja & 2 & Tietê-Batalha \\
\hline G9-R2 & $\begin{array}{l}\text { Matão, Faz. Cambuhy } \\
21^{\circ} 37^{\prime} 39^{\prime \prime S} \text { e } 48^{\circ} 32^{\prime} 12^{\prime \prime} \mathrm{W}\end{array}$ & interior & $39.128,9$ & $2.189,6$ & 2,36 & laranja & 1 & Tietê-Batalha \\
\hline
\end{tabular}

a. Riacho não amostrado (seco);

b. Conforme Laurance \& Yensen (1991): P/200[(pi.AT)0,5], onde P: perímetro do fragmento (em metros) e AT: área total (em hectares);

c. Conforme Stralhler (1957);

d. Unidades de Gerenciamento de Recursos Hídricos.

sombreamento: aberto, parcialmente sombreado, sombreado e fortemente sombreado (modificado de De Nicola et al. 1992). Para tal classificação foi feita uma estimativa visual, sendo considerados: abertos, os riachos que apresentaram ausência de mata ciliar; parcialmente sombreados, aqueles com mata ciliar baixa ou em apenas uma das margens; sombreados, aqueles com a mata ciliar cobrindo o trecho por inteiro e apenas um extrato arbóreo; e fortemente sombreados, os riachos que apresentaram mais de um extrato arbóreo cobrindo o trecho por completo.

As amostragens foram realizadas no intervalo de junho a agosto de 2007 e de 2008 , dentro do período típico de maior abundância e riqueza de espécies de macroalgas em ambientes lóticos da região (Necchi Jr. \& Pascoaloto 1993, Branco \& Necchi Jr. 1997), por 


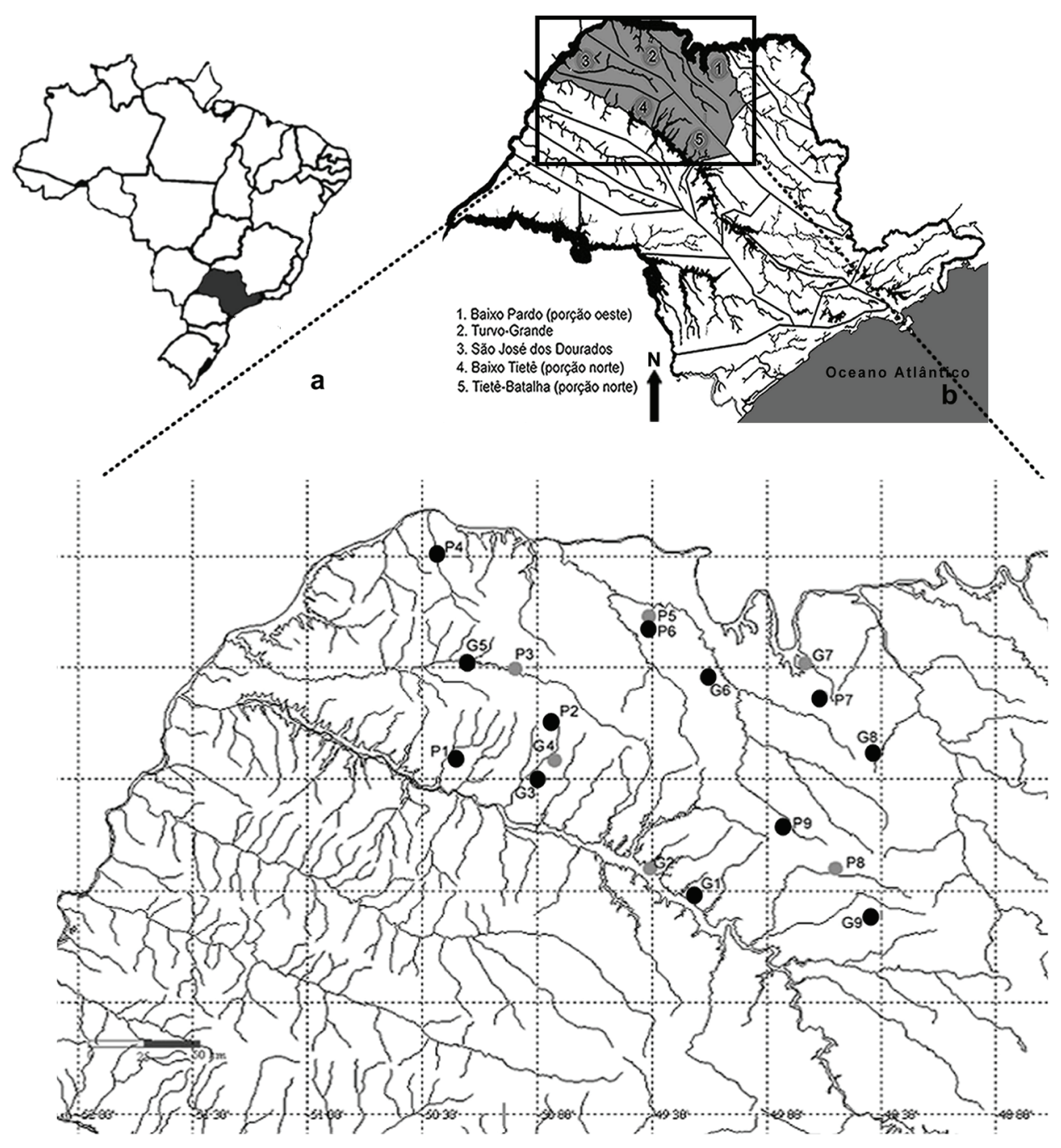

Figura 1. a. Mapa do Brasil destacando o Estado de São Paulo. b. Mapa do Estado de São Paulo destacando a região noroeste conforme definida neste estudo, evidenciando as Unidades de Gerenciamento de Recursos Hídricos e os fragmentos amostrados (pontos em negrito) e não amostrados (pontos em cinza).

Figure 1. a. Map of Brazil showing São Paulo State. b. Map of São Paulo State showing the northwest region with the Units of Water Resources Management and the forest fragments sampled (boldface dots) and unsampled (gray dots).

meio da técnica de quadrado associada à de transeção de linha (Necchi Jr. 2004). Foi delimitado um trecho de 10 metros de extensão, subdividido em intervalos iguais de um metro. Duas unidades amostrais foram distribuídas de forma equidistante em relação à largura sobre o leito do rio, a cada intervalo de um metro de comprimento, totalizando 20 unidades no trecho todo. Foi adotado o conceito de macroalga proposto por Sheath \& Cole (1992).

A preservação dos espécimes foi feita em solução de formaldeído 4\% (Necchi Jr. 2004). Os procedimentos para análise e identificação foram aqueles descritos nos trabalhos taxonômicos de macroalgas lóticas citados anteriormente. Conjuntos representativos de espécimes-testemunho foram incorporados no Herbário SJRP (Holmgren \& Holmgren 2011). Os espécimes amostrados foram posteriormente identificados em laboratório, sempre que possível, até o nível espécie. Para classificação dos filos foi adotado o sistema de Graham et al. (2009). Para todos os táxons foram feitas ilustrações (fotomicrografias), usando câmera de vídeo digital Leica DFC 320, e software de captura e análise de imagem (Leica IM50), acoplado ao microscópio Leica DM 5.000. Foram apresentadas, ainda, descrições e, quando necessário, considerações taxonômicas, 
além dos pontos de ocorrência de cada táxon com as respectivas características físico-químicas da água. Em relação ao tamanho das células foi utilizada a sigla $\mathrm{C} / \mathrm{D}$, que representa o comprimento pelo diâmetro celular.

\section{Resultados}

\section{Chlorophyta}

Microspora floccosa (Vauch.) Thur. Ann. Sci. Nat., ser. Bot., (3)14: 214-260, pls 16-31. 1850.

Figuras 2-3

Emaranhado de filamentos, verde-amarelados, filamentos levemente constritos, 10,5-13,0 $\mu$ m diâm.; células cilíndricas ou doliformes, (9,5-)11,0-17,5 $\mu \mathrm{m}$ compr., 9,0-11,0 $\mu \mathrm{m}$ diâm., C/D 0,8-2,3; estruturas em $\mathrm{H}$ claramente visíveis; cloroplastos laminares, cobrindo a maior parte da célula.

Condições ambientais: $(\mathrm{n}=2)$ : Temperatura $22,1-22,5{ }^{\circ} \mathrm{C}(\mathrm{X}=22,3 \pm 0,2)$; Condutividade 6,0-10,0 $\mu \mathrm{S} \mathrm{cm} \mathrm{cm}^{-1}(\mathrm{X}=7,3 \pm 2,3)$; Turbidez 5,0-15,0 UNT ( $X=11,3 \pm 5,5)$; Velocidade da correnteza 1,6-70,2 $\mathrm{cm} \mathrm{s}^{-1}(\mathrm{X}=32,1 \pm 17,6) ; \mathrm{pH}$ 6,7-6,9 ( $\mathrm{X}=6,8 \pm 0,1)$; Oxigênio dissolvido $3,2-3,9 \mathrm{mg} \mathrm{L}^{-1}(\mathrm{X}=3,6 \pm 0,4)$; Profundidade $5,0-25,0 \mathrm{~cm}(\mathrm{X}=11,3 \pm 4,7)$.

Material examinado: BRASIL. São PaUlo: Santo Antonio do Aracanguá, Vicentópolis, Rancho Alegre, 17-VIII-2007, F.V.R. Almeida \& O. Necchi Jr. s.n. (SJRP29701); Barretos, 17-VIII-2007, F.V.R. Almeida \& O. Necchi Jr. s.n. (SJRP29709).

Microspora quadrata Haz., Mem. Torrey Bot. Cl. 11: 178.1887.

\section{Figuras 4-5}

Emaranhado de filamentos, verde-amarelados, filamentos levemente constritos, 7,5-9,5 $\mu \mathrm{m}$ diâm.; células quadráticas, cilíndricas, ou levemente doliformes, 8,5-12,0 $\mu \mathrm{m}$ compr., 6,5-8,0 $\mu \mathrm{m}$ diâm., C/D 0,8-1,5; estruturas em $\mathrm{H}$ claramente visíveis; cloroplastos laminares, cobrindo a maior parte da célula.

Condições ambientais: $(\mathrm{n}=1)$ : Temperatura $22,1-22,5{ }^{\circ} \mathrm{C}(\mathrm{X}=22,3 \pm 0,2)$; Condutividade $6,0-10,0 \mu \mathrm{S} \mathrm{cm} \mathrm{cm}^{-1}(\mathrm{X}=7,3 \pm 2,3)$; Turbidez $5,0-15,0$ UNT $(X=11,3 \pm 5,5)$; Velocidade da correnteza 1,6-70,2 $\mathrm{cm} \mathrm{s}^{-1}(\mathrm{X}=32,1 \pm 17,6) ; \mathrm{pH}$ 6,7-6,9 ( $\mathrm{X}=6,8 \pm 0,1)$; Oxigênio dissolvido $3,2-3,9 \mathrm{mg} \mathrm{L}^{-1}(\mathrm{X}=3,6 \pm 0,4)$; Profundidade $8,0-25,0 \mathrm{~cm}(\mathrm{X}=11,3 \pm 4,7)$.

Material examinado: BRASIL. São Paulo: Santo Antonio do Aracanguá, Vicentópolis, Rancho Alegre, 17-VIII-2007, F.V.R. Almeida \& O. Necchi Jr. s.n. (SJRP29701)

Nitella rosa-mariae Picelli Vic., Flora ficológica do Estado de São Paulo - Charophyceae, 5: 64-65. 2004. Figuras 6-8

Plantas monóicas, ecorticadas, desprovidas de incrustação calcária; verticilos estéreis bem desenvolvidos; verticilos férteis reduzidos, formando capítulos desprovidos de muco; capítulos pedunculados; râmulos verticilados dimórficos 3-4 furcados; dáctilos 3-5, 2-3 celulados; gametângios pedunculados, presentes na primeira furcação dos râmulos conjuntos ou sejuntos; núculas 1-2 por nó, 275-500 $\mu \mathrm{m}$ compr., 200-400 $\mu \mathrm{m}$ diâm.; glóbulos 150-225 $\mu \mathrm{m}$ diâm.

Condições ambientais: $(\mathrm{n}=1)$ : Temperatura $22,1-22,5{ }^{\circ} \mathrm{C}(\mathrm{X}=22,3 \pm 0,2)$; Condutividade $6,0-10,0 \mu \mathrm{S} \mathrm{cm}^{-1}(\mathrm{X}=7,3 \pm 2,3)$; Turbidez 5,0-15,0 UNT $(X=11,3 \pm 5,5)$; Velocidade da correnteza 1,6-70,2 $\mathrm{cm} \mathrm{s}^{-1}(\mathrm{X}=32,1 \pm 17,6) ; \mathrm{pH}$ 6,7-6,9 ( $\mathrm{X}=6,8 \pm 0,1)$; Oxigênio dissolvido 3,2-3,9 $\mathrm{mg} \mathrm{L}^{-1}(\mathrm{X}=3,6 \pm 0,4)$; Profundidade $5,0-25,0 \mathrm{~cm}(\mathrm{X}=11,3 \pm 4,7)$.

Material examinado: BRASIL. São Paulo: Santo Antonio do Aracanguá, Vicentópolis, Rancho Alegre, 17-VIII-2007, F.V.R. Almeida \& O. Necchi Jr. s.n. (SJRP29699).

Este táxon já foi registrado anteriormente para o Estado de São Paulo, inclusive para a região noroeste (Vieira et al. 2002, como Nitella sp.).

Nitella subglomerata A. Braun. Monatsberichte der Deutschen Akademie der Wissenchaft zu Berlin 1858: 356. 1859.

Figuras 9-11

Plantas monóicas, ecorticadas, desprovidas de incrustação calcária; verticilos estéreis bem desenvolvidos, verticilos férteis reduzidos, formando capítulos frouxos e desprovidos de muco; capítulos pedunculados; râmulos verticilados estéreis 7-9, 
1-furcado; dáctilos 3-5, 1-celulado, acuminados; gametângios sésseis, raramente pedunculados, presentes na primeira furcação dos râmulos, conjuntos ou sejuntos, dentro ou fora dos capítulos; núculas 1-2 por nó;

Condições ambientais: $(\mathrm{n}=1)$ : Temperatura $18,8-18,9{ }^{\circ} \mathrm{C}(\mathrm{X}=18,8 \pm 0,1)$; Condutividade $26,0 \mu \mathrm{S} \mathrm{cm}^{-1}(\mathrm{X}=26,0 \pm 0,0)$; Turbidez 23,0 UNT $(\mathrm{X}=23,0 \pm 0,0)$; Velocidade da correnteza 9,1-22,5 $\mathrm{cm} \mathrm{s}^{-1}$ $(\mathrm{X}=15,7 \pm 6,7)$; pH 6,7-6,9 $(\mathrm{X}=6,8 \pm 0,1)$; Oxigênio dissolvido 5,6 $\mathrm{mg} \mathrm{L}^{-1}(\mathrm{X}=5,6 \pm 0,0)$; Profundidade $6,0-33,0 \mathrm{~cm}(\mathrm{X}=14,7 \pm 15,9)$.

Material examinado: BRASIL. SÃo PAUlo: Barretos, 17-VI-2007, F.V.R. Almeida s.n. (SJRP29711).

\section{Oedogonium sp.1}

Figura 12

Células cilíndricas, 18,5-32,0 $\mu \mathrm{m}$ compr., 5,6-8,0 $\mu \mathrm{m}$ diâm., C/D 2,9-4,7.

Condições ambientais: $(\mathrm{n}=1)$ : Temperatura 20,5-20,8 ${ }^{\circ} \mathrm{C}(\mathrm{X}=20,6 \pm 0,2)$; Condutividade $1,0-3,0 \mu \mathrm{S} \mathrm{cm} \mathrm{cm}^{-1}(\mathrm{X}=2,0 \pm 1,0)$; Turbidez 14,0-15,0 UNT $(X=14,3 \pm 0,6)$; Velocidade da correnteza $0,0-63,4 \mathrm{~cm} \mathrm{~s}^{-1}(X=18,9 \pm 22,5)$; pH 6,1-6,2 (X $=6,2 \pm 0,1)$; Oxigênio dissolvido 7,3-7,9 $\mathrm{mg} \mathrm{L}^{-1}(\mathrm{X}=7,7 \pm 0,3)$; Profundidade $2,0-18,0 \mathrm{~cm}(\mathrm{X}=10,2 \pm 4,7)$.

Material examinado: BRASIL. São PAULo: Planalto, Fazenda Taperão, 11-VII-2007, F.V.R. Almeida s.n. (SJRP29702).

Foram encontrados apenas espécimes estéreis, o que impossibilitou a identificação em nível específico. Dessa forma, os espécimes foram divididos em 3 "grupos vegetativos" para permitir referência futura a essas populações em estudos ecológicos e taxonômicos da região. Essa divisão foi baseada no diâmetro das células: Oedogonium sp.1 (5-8 $\mu \mathrm{m})$, Oedogonium sp.2 (11-16 $\mu \mathrm{m})$, e Oedogonium sp.3 (20-34 $\mu \mathrm{m})$. Esses grupos não têm valor taxonômico formal.

\section{Oedogonium sp.2}

Figura 13

Células cilíndricas, 32,0-48,0(-56,0) $\mu \mathrm{m}$ compr., 11,0-16,0 $\mu \mathrm{m}$ diâm., C/D 1,8-4,0, com grânulos citoplasmáticos bem evidentes.
Condições ambientais: $(\mathrm{n}=2)$ : Temperatura $22,1-22,5{ }^{\circ} \mathrm{C}(\mathrm{X}=22,3 \pm 0,2)$; Condutividade $6,0-10,0 \mu \mathrm{S} \mathrm{cm} \mathrm{cm}^{-1}(\mathrm{X}=7,3 \pm 2,3)$; Turbidez $5,0-15,0$ UNT $(X=11,3 \pm 5,5)$; Velocidade da correnteza 1,6-70,2 $\mathrm{cm} \mathrm{s}^{-1}(\mathrm{X}=30,0 \pm 17,4) ; \mathrm{pH}$ 6,7-6,9 ( $\mathrm{X}=6,8 \pm 0,1)$; Oxigênio dissolvido 3,2-3,9 $\mathrm{mg} \mathrm{L}^{-1}(\mathrm{X}=4,8 \pm 0,1)$; Profundidade $5,0-33,0 \mathrm{~cm}(\mathrm{X}=11,8 \pm 6,6)$.

Material examinado: BRASIL. São PAUlo: Santo Antonio do Aracanguá, Vicentópolis, Rancho Alegre, 17-VIII-2007, F.V.R. Almeida \& O. Necchi Jr. s.n. (SJRP29700). BRASIL. São Paulo: Barretos, 17-VI-2007, F.V.R. Almeida s.n. (SJRP29700).

\section{Oedogonium sp.3}

Figura 14

Células cilíndricas, 32,0-50,0 $\mu \mathrm{m}$ compr., 20,0-32,0(-34,0) $\mu \mathrm{m}$ diâm., C/D (0,8-)1,1-4,5(-4,8), levemente constritas nos septos.

Condições ambientais: $(\mathrm{n}=2)$ : Temperatura 16,3-18,9 ${ }^{\circ} \mathrm{C}(\mathrm{X}=18,0 \pm 1,5)$; Condutividade 26,0-30,0 $\mu \mathrm{S} \mathrm{cm}^{-1}(\mathrm{X}=27,3 \pm 2,3)$; Turbidez 23,0-25,0 UNT ( $\mathrm{X}=23,7 \pm 1,2)$; Velocidade da correnteza $0,0-38,5 \mathrm{~cm} \mathrm{~s}^{-1}(X=17,8-13,1)$; $\mathrm{pH} 7,4-7,5(\mathrm{X}=7,4 \pm 0,1)$; Oxigênio dissolvido 4,8-6,4 $\mathrm{mg} \mathrm{L}^{-1}(\mathrm{X}=5,3 \pm 0,5)$; Profundidade $9,1-38,5 \mathrm{~cm}(\mathrm{X}=23,9 \pm 10,6)$.

Material examinado: BRASIL. São PAULO: Bebedouro, Fazenda Córrego dos Bois, 17-VI-2007, F.V.R. Almeida s.n. (SJRP29711); Barretos, 17-VI-2007, F.V.R. Almeida s.n. (SJRP29706).

\section{Spirogyra sp.1}

Figura 15

Filamentos emaranhados, células cilíndricas,

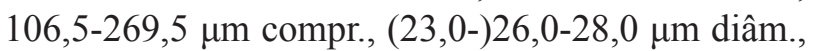
C/D 2,1-3,9.

Condições ambientais: $(\mathrm{n}=1)$ : Temperatura $18,9-18,8{ }^{\circ} \mathrm{C}(\mathrm{X}=18,8 \pm 0,1)$; Condutividade $26,0 \mu \mathrm{S} \mathrm{cm}^{-1}(\mathrm{X}=26,0 \pm 0,0)$; Turbidez 23,0 UNT $(\mathrm{X}=23,0 \pm 0,0)$; Velocidade da correnteza 9,1-22,5 $\mathrm{cm} \mathrm{s}^{-1}$ $(\mathrm{X}=15,7 \pm 6,7) ; \mathrm{pH} 6,7-6,9(\mathrm{X}=6,8 \pm 0,1)$; Oxigênio dissolvido 5,6 $\mathrm{mg} \mathrm{L}^{-1}(\mathrm{X}=5,6 \pm 0,0)$; Profundidade $6,0-33,0(X=14,7 \pm 15,9)$.

Material examinado: BRASIL. São Paulo: Barretos, 17-VI-2007, F.V.R. Almeida s.n. (SJRP29700). 
Da mesma forma que em Oedogonium spp., foram encontrados apenas espécimes estéreis nos pontos de amostragem, que foram divididos em dois "grupos vegetativos", baseados no diâmetro das células: Spirogyra sp.1 (20,0-30,0 $\mu \mathrm{m})$ e Spirogyra sp.2 $(45,0-60,0 \mu \mathrm{m})$.

\section{Spirogyra sp.2}

Figura 16

Filamentos emaranhados, células cilíndricas,

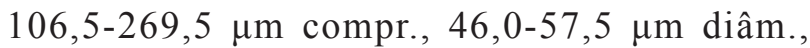
C/D 1,9-4,9.

Condições ambientais: $(\mathrm{n}=1)$ : Temperatura $22,1-22,5{ }^{\circ} \mathrm{C}(\mathrm{X}=22,3 \pm 0,2)$; Condutividade $6,0-10,0 \mu \mathrm{S} \mathrm{cm} \mathrm{cm}^{-1}(\mathrm{X}=7,3 \pm 2,3)$; Turbidez $5,0-15,0$ UNT $(X=11,3 \pm 5,5)$; Velocidade da correnteza $11,6-70,2 \mathrm{~cm} \mathrm{~s}^{-1}(\mathrm{X}=30,0 \pm 17,4)$; $\mathrm{pH}$ 6,7-6,9 $(\mathrm{X}=6,8 \pm 0,1)$; Oxigênio dissolvido 3,2-3,9 $\mathrm{mg} \mathrm{L}^{-1}(\mathrm{X}=4,8 \pm 0,1)$; Profundidade $5,0-33,0 \mathrm{~cm}(\mathrm{X}=11,8 \pm 6,6)$.

Material examinado: BRASIL. São PaUlo: Santo Antonio do Aracanguá, Vicentópolis, Rancho Alegre, 17-VIII-2007, F.V.R. Almeida \& O. Necchi Jr. s.n. (SJRP29700).

\section{Cyanophyta}

Geitlerinema splendidum (Gom.) Anag., Pl. Syst. Evol. 164: 33-46. 1989.

Figuras 17-18

Tricomas emaranhados, retos ou flexuosos, distintamente atenuados, não constritos, curvos no ápice; células 3,0-5,0(-7,0) $\mu \mathrm{m}$ compr., 1,6-2,5 $\mu \mathrm{m}$ diâm., C/D 1,2-3,2(-3,5); conteúdo celular verde-azulado, homogêneo, septos granulados, 1 ou 2 grânulos de cada lado; células apicais capitadas.

Condições ambientais: $(\mathrm{n}=3)$ : Temperatura 20,5-25, $1{ }^{\circ} \mathrm{C}(\mathrm{X}=22,3 \pm 2,3)$; Condutividade $1,0-22,0 \mu \mathrm{S} \mathrm{cm} \mathrm{cm}^{-1}(\mathrm{X}=9,2 \pm 10,1)$; Turbidez 14,0-15,0 UNT $(X=14,4 \pm 0,5)$; Velocidade da correnteza $0,0-63,4 \mathrm{~cm} \mathrm{~s}^{-1}(X=13,8 \pm 17,0)$; $\mathrm{pH}$ 6,1-7,2 (X $=6,5 \pm 0,5)$; Oxigênio dissolvido 4,8-7,9 $\mathrm{mg} \mathrm{L}^{-1}(\mathrm{X}=6,5 \pm 1,6)$; Profundidade $2,0-25,0 \mathrm{~cm}(\mathrm{X}=9,6 \pm 5,9)$.

Material examinado: BRASIL. São PAULo: Planalto, Fazenda Taperão, 11-VII-2007, F.V.R. Almeida s.n. (SJRP29703); Palestina, 15-VII-2008, F.V.R. Almeida s.n. (SJRP29702).
Jaaginema homogeneum (Frémy) Anag. \& Kom., Algolog. Stud. 50-53: 396. 1988.

Figura 19

Filamentos levemente curvados, geralmente solitários, às vezes formando tufos, não atenuados nas extremidades; bainha na maioria das vezes ausente, raramente como mucilagem difusa e incolor ao redor dos tricomas; tricomas não constritos; células mais longas que largas, (4,0-)4,8-7,2 $\mu \mathrm{m}$ compr., 3,2-3,6(-4,0) بm diâm., C/D (1,2-)1,5-2,2; conteúdo celular verde-azulado, com grânulos protoplasmáticos; célula apical cilíndrico-arredondada ou levemente cônico-arredondada, sem caliptra.

Condições ambientais: $(\mathrm{n}=1)$ : Temperatura $17,6-17,7{ }^{\circ} \mathrm{C}(\mathrm{X}=17,6 \pm 0,1)$; Condutividade $40,0-78,0 \mu \mathrm{S} \mathrm{cm}^{-1}(\mathrm{X}=59,0 \pm 26,9)$; Turbidez $11,0 \mathrm{UNT}$ $(\mathrm{X}=11,0 \pm 0,0)$; Velocidade de correnteza $2,0-85,8 \mathrm{~cm}$ $\mathrm{s}^{-1}(\mathrm{X}=57,8 \pm 19,5) ; \mathrm{pH} 7,5-7,6(\mathrm{X}=7,5 \pm 0,0)$; Oxigênio dissolvido 5,2-5,8 $\mathrm{mg} \mathrm{L}^{-1}(\mathrm{X}=5,5 \pm 0,4)$; Profundidade 8,0-208 $(\mathrm{X}=26,0 \pm 44,4)$;

Material examinado: BRASIL. São Paulo: Nova Granada, Fazenda São João, 16-VII-2008, F.V.R. Almeida s.n. (SJRP29704).

Microcoleus subtorulosus Gom., Ann. Sci. Nat. Bot. (7)15: 360. 1892.

Figura 20

Filamentos emaranhados, azul-acinzentado, não ramificado, 9,0-20,0 $\mu \mathrm{m}$ diâm.; bainha homogênea, levemente amarelada, não lamelada; 1-3 tricomas por filamento, constritos nos septos; células geralmente mais longas do que largas ou isodiamétricas, (4,0-)5,0-9,0 $\mu \mathrm{m}$ compr., (4,5-)5,0-6,5 $\mu \mathrm{m}$ diâm., C/D 0,8-1,6(-1,8); conteúdo celular verde-azulado, com grânulos protoplasmáticos; célula apical predominantemente cônico-arredondada ou cilíndrico-arredondada.

Condições ambientais: $(\mathrm{n}=1)$ : Temperatura $14,3-14,6{ }^{\circ} \mathrm{C}(\mathrm{X}=14,5 \pm 0,2)$; Condutividade $37,0-41,0 \mu \mathrm{S} \mathrm{cm}^{-1}(\mathrm{X}=39,3 \pm 2,1)$; Turbidez 2,0 U NT $(X=2,0 \pm 0,0)$; Velocidade da correnteza $1,6-120,6 \mathrm{~cm} \mathrm{~s}^{-1}(\mathrm{X}=30,4 \pm 29,4) ; \mathrm{pH} \mathrm{6,54-7,44}$ $(\mathrm{X}=7,1 \pm 0,5)$; Oxigênio dissolvido $5,9-6,6 \mathrm{mg} \mathrm{L}^{-1}$ $(X=6,2 \pm 0,4)$; Profundidade 7,0-27,0 $\mathrm{cm}$ $(X=15,2-5,5)$.

Material examinado: BRASIL. São Paulo: São Francisco de Iracema, Fazenda São Francisco, 19-VII-2007, F.V.R. Almeida s.n. (SJRP29804). 


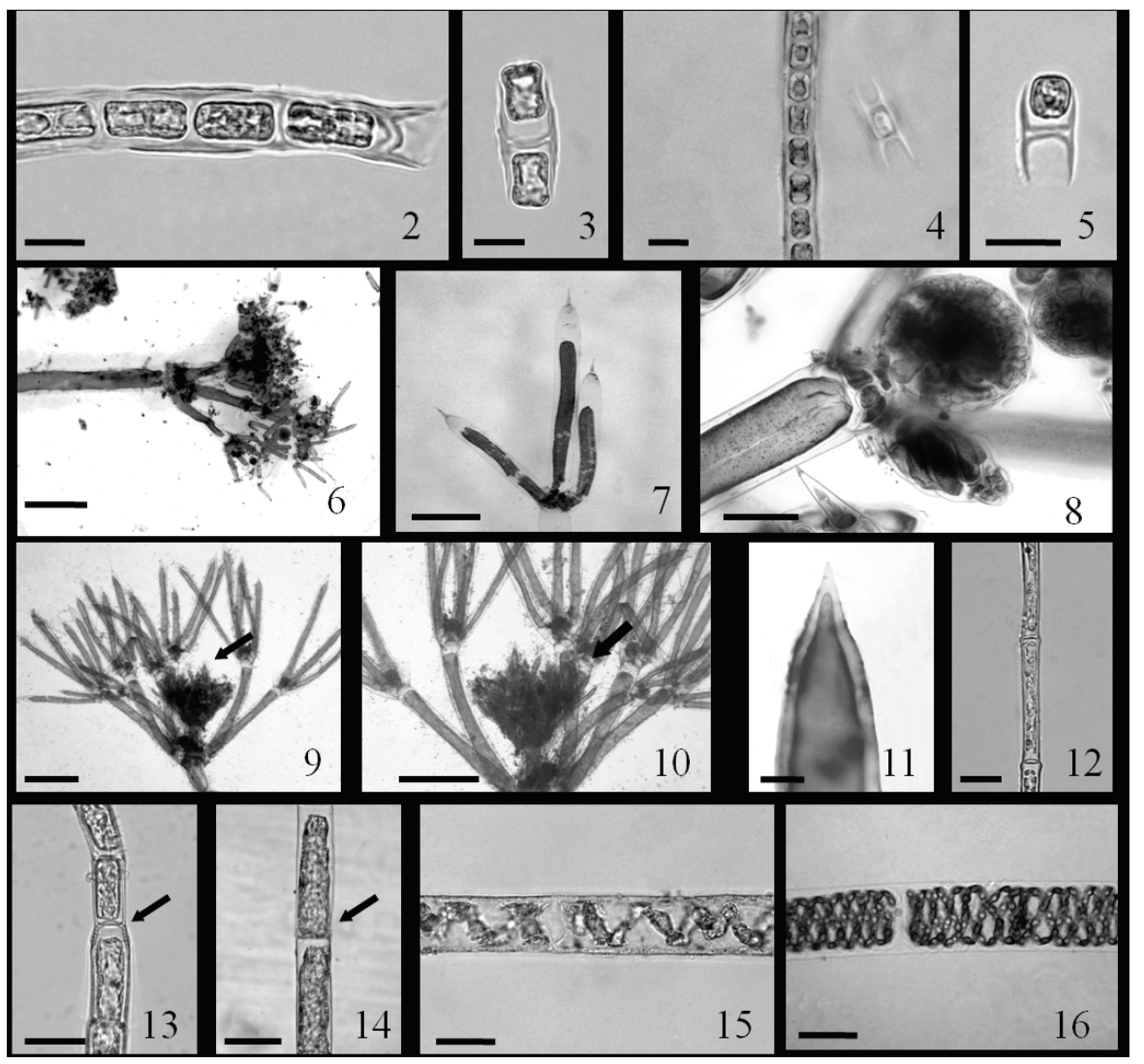

Figuras 2-16. Fotomicrografias das espécies de Chlorophyta descritas. 2-3. Microspora floccosa. 2. Ápice do filamento evidenciando peça em H (seta). 3. Detalhe da parede em forma de H. 4-5. Microspora quadrata. 5. Detalhe da parede em forma de H. 6-8. Nitella rosamariae. 6. Aspecto geral do capítulo. 7. Dáctilos 2-celulados. 8. Detalhe mostrando glóbulo (seta) e núcula pedunculados (ponta de seta). 9-11. Nitella subglomerata. 9-10. Ápice da planta evidenciando os capítulos frouxos (setas). 11. Dáctilo unicelulado acuminado. 12. Oedogonium sp.1. 13. Oedogonium sp.2. 14. Oedogonium sp.3. 13-14. Setas evidenciando os anéis de crescimento. 15 . Spirogyra sp.1. 16. Spirogyra sp.2. (Escalas: Figuras 2-5, $12=10 \mu \mathrm{m} ; 13,15=25 \mu \mathrm{m} ; 8,14,16=50 \mu \mathrm{m} ; 11=100 \mu \mathrm{m} ; 7=0,5 \mathrm{~mm} ; 6,9,10=1 \mathrm{~mm}$ ).

Figures 2-16. Photomicrographs of Chlorophyta species. 2-3. Microspora floccosa. 2. Filament apex showing H-shaped wall (arrow). 3. Detail of H-shaped wall. 4-5. Microspora quadrata. 5. Detail of H-shaped wall. 6-8. Nitella rosamariae. 6. General view of the head. 7. 2-celled dactyl. 8. Detail of stalked globule (arow) and nucule (arrowhead). 9-11. Nitella subglomerata.9-10. Apices of filaments showing loosely arranged heads (arrows). 11. One-celled acuminated dactyl. 12. Oedogonium sp.1. 13. Oedogonium sp.2. 14. Oedogonium sp.3. 13-14. Detail of filaments showing striaes (arrows). 15. Spirogyra sp.1. 16. Spirogyra sp.2. (Scale bars: Figures. 2-5, $12=10 \mu$ m; 13 , $15=25 \mu \mathrm{m} ; 8,14,16=50 \mu \mathrm{m} ; 11=100 \mu \mathrm{m} ; 7=0,5 \mathrm{~mm} ; 6,9,10=1 \mathrm{~mm})$.

As amostras analisadas apresentaram menor proporção no diâmetro do filamento, notadamente grande redução da bainha, comparando-se com material de estudos realizados no Estado de São Paulo (San’t Anna \& Azevedo 1995, Necchi Jr. et al. 1997, Branco et al. 1999, 15,0-115,0 $\mu \mathrm{m}$ ), ocorreu também com Trichocoleus sociatus, que foi coletado no mesmo riacho. Tais observações sugerem possível interferência do hábitat em sua morfologia.

Oscilatoria tenuis Ag. ex Gom., Ann. Sci. Nat. 16: 220.1892.

Figura 21

Tricomas solitários, retos, não atenuados ou levemente atenuados no ápice, levemente constritos; células mais curtas do que largas, $2,0-4,0 \mu \mathrm{m}$ compr., 11,0-12,0 $\mu \mathrm{m}$ diâm., C/D 0,2-0,3; conteúdo celular verde-azulado, com grânulos protoplasmáticos; célula apical cilíndrico-arredondada.

Condições ambientais: $(\mathrm{n}=1)$ : Temperatura $22,3-22,6{ }^{\circ} \mathrm{C}(\mathrm{X}=22,4 \pm 0,2)$; Condutividade $6,0-8,0 \mu \mathrm{S} \mathrm{cm}^{-1}(\mathrm{X}=7,0 \pm 1,4)$; Turbidez 2,0 UNT $(\mathrm{X}=2,0 \pm 0,0)$; Velocidade da correnteza $0,0-1,7 \mathrm{~cm} \mathrm{~s}^{-1}$ $(\mathrm{X}=0,2 \pm 0,6) ; \mathrm{pH} 6,6-7,4(\mathrm{X}=7,0 \pm 0,5)$; Oxigênio dissolvido 4,4-4,7 mg L-1 $(\mathrm{X}=4,6 \pm 0,2)$; Profundidade $4,0-25,0 \mathrm{~cm}(\mathrm{X}=13,0 \pm 7,5)$.

Material examinado: BRASIL. SÃo PAULo: Palestina, 19-VII-2007, F.V.R. Almeida s.n. (SJRP29702). 
Phormidium retzii (C. Ag.) Gom., Ann. Sci. Nat. Bot. (7)16: 175. 1892.

Figura 22

Filamentos em feixes emaranhados e prostrados, com bainha delgada, hialina, não lamelada, 6,0-9,0 $\mu \mathrm{m}$ diâm.; tricomas não constritos,

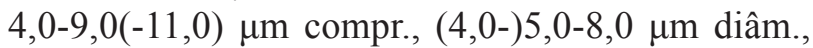
C/D 0,6-2,0; conteúdo celular verde-azulado, com grânulos protoplasmáticos; célula apical cilíndrica ou cônico arredondada; hormogônios e necrídeos presentes.

Condições ambientais: $(\mathrm{n}=6)$ : Temperatura $16,3-25,1{ }^{\circ} \mathrm{C}(\mathrm{X}=20,0 \pm 2,7)$; Condutividade $1,0-78,0 \mu \mathrm{S} \mathrm{cm} \mathrm{cm}^{-1}(\mathrm{X}=16,8 \pm 19,2)$; Turbidez 4,0-51,0 UNT $(\mathrm{X}=16,2 \pm 12,1)$; Velocidade da correnteza $0,0-85,8 \mathrm{~cm} \mathrm{~s}^{-1}(\mathrm{X}=32,8 \pm 21,2)$; pH 6,1-7,6 (X = 6,8 $\pm 0,4)$; Oxigênio dissolvido $3,1-7,9 \mathrm{mg} \mathrm{L}^{-1}(\mathrm{X}=5,6 \pm 1,4)$; Profundidade 2,0-208,0 cm (X=16,4-21,8).

Material examinado: BRASIL. São PAULo: Santo Antonio do Aracanguá, Vicentópolis, Rancho Alegre, 17-VIII-2007, F.V.R. Almeida \& O. Necchi Jr. s.n. (SJRP29804); Novo Horizonte, Fazenda Serrinha, 14-VII-2007, F.V.R. Almeida s.n. (SJRP29804); Nova Granada, Fazenda São João, 18-VI-2008, F.V.R. Almeida s.n. (SJRP29705); Bebedouro, Fazenda Córrego dos Bois, 17-VI-2007, F.V.R. Almeida s.n. (SJRP29707); Bebedouro, Fazenda Córrego dos Bois, 4-VII-2007, F.V.R. Almeida s.n. (SJRP29710).

A amostra analisada apresentou considerável variabilidade nas características morfométricas, sugerindo tratar-se de um complexo de diferentes espécies (Geitler 1932, Branco et al. 1999).

Phormidium cf. retzii (C. Ag.) Gom., Ann. Sci. Nat. Bot. (7)16: 175. 1892.

Figura 23

Filamentos em feixes emaranhados e prostrados, com bainha ausente ou bastante delgada, não lamelada, 6,0-6,5 $\mu \mathrm{m}$ diâm.; tricomas não constritos, 5,0-9,0 (-10,0) $\mu \mathrm{m}$ compr., 5,0-6,0 $\mu \mathrm{m}$ diâm., C/D (1,0-1,5)(-1,7); conteúdo celular verde-azulado, com grânulos protoplasmáticos; célula apical cilíndrica ou cônico arredondada. Hormogônios e necrídeos não observados.

Condições ambientais: $(\mathrm{n}=1)$ : Temperatura 22,3-22,6 ${ }^{\circ} \mathrm{C}(\mathrm{X}=22,4 \pm 0,2)$; Condutividade
$6,0-8,0 \mu \mathrm{S} \mathrm{cm}^{-1}(\mathrm{X}=7,0 \pm 1,4)$; Turbidez 2,0 UNT $(\mathrm{X}=2,0 \pm 0,0)$; Velocidade da correnteza $0,0-1,7 \mathrm{~cm} \mathrm{~s}^{-1}$ $(\mathrm{X}=0,2 \pm 0,6)$; pH 6,6-7,4 $(\mathrm{X}=7,0 \pm 0,5)$; Oxigênio dissolvido 4,4-4,7 $\mathrm{mg} \mathrm{L}^{-1}(\mathrm{X}=4,6 \pm 0,2)$; Profundidade $4,0-25,0 \mathrm{~cm}(\mathrm{X}=13,0 \pm 7,4)$.

Material examinado: BRASIL. São PAULo: Palestina, 19-VII-2007, F.V.R. Almeida s.n. (SJRP29702).

A amostra analisada foi identificada como Phormidium cf. retzii por ter apresentado diferença no comprimento das células, quadráticas ou mais longas que largas, e nunca mais curtas.

Scytonema arcangeli Born. \& Flah., Ann. Sci. Nat. Bot. (7) 5: 92.1886.

Figuras 24-26

Filamentos agrupados formando tufos eretos, verde-azulados, 16,0-20,0 $\mu \mathrm{m}$ diâm.; ramificações falsas duplas abundantes e ramificações simples ocasionais; bainha hialina, geralmente incolor, às vezes amarelada, sem lamelação; tricomas predominantemente constritos, sem área meristemática definida, dispersas ao longo do filamento; células em sua maioria mais curtas do que largas, $8,0-12,0 \mu \mathrm{m}$ compr., 12,0-16,0 $\mu \mathrm{m}$ diâm., C/D 0,5-0,8 (1,0); heterócitos

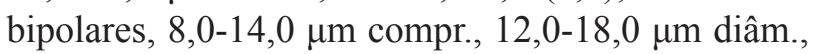
C/D 0,5-1,0 (1,2); hormogônios presentes.

Condições ambientais: $(\mathrm{n}=1)$ : Temperatura 20,5-20,8 ${ }^{\circ} \mathrm{C}(\mathrm{X}=20,6 \pm 0,2)$; Condutividade $1,0-3,0 \mu \mathrm{S} \mathrm{cm}^{-1}(\mathrm{X}=2,0 \pm 1,0)$; Turbidez 14,0-15,0 UNT $(\mathrm{X}=14,3 \pm 0,6)$; Velocidade da correnteza $0,0-63,4 \mathrm{~cm} \mathrm{~s}^{-1}(\mathrm{X}=18,9 \pm 22,5) ; \mathrm{pH} \mathrm{6,1-6,2}$ $(\mathrm{X}=6,2 \pm 0,1)$; Oxigênio dissolvido 7,3-7,9 $\mathrm{mg} \mathrm{L}^{-1}$ $(X=7,7 \pm 0,3) ;$ Profundidade 2,0-18,0 cm $(X=10,2 \pm 4,7)$.

Material examinado: BRASIL. SÃo PAULO: Planalto, Fazenda Taperão, 11-VII-2007, F.V.R. Almeida s.n. (SJRP29703).

Trichocoleus sociatus (W. West \& G.S. West) Anag., Preslia 73: 369. 2001.

Figuras 27-28

Filamentos não ramificados, longos, 11,0-30,0 $\mu \mathrm{m}$ diâm.; bainha firme, transparente ou castanha, aberta na extremidade, contendo 10-13 tricomas, emaranhados ou torcidos em espiral; tricomas verde-azulados, às vezes acinzentados, constritos nos septos, não granulados; células geralmente mais longas do que 
largas, 4,5-8,0 $\mu \mathrm{m}$ compr., (1,5-)2,5-3,5 $\mu \mathrm{m}$ diâm., C/D 1,0-3,0; célula apical cônico-arredondada, alongada, sem caliptra.

Condições ambientais: $(\mathrm{n}=1)$ : Temperatura $14,3-14,6{ }^{\circ} \mathrm{C}(\mathrm{X}=14,5 \pm 0,2)$; Condutividade $37,0-41,0 \mu \mathrm{S} \mathrm{cm}$-1 $\left.^{-1}=39,3 \pm 2,1\right)$; Turbidez 2,0 UNT $(X=2,0 \pm 0,0)$; Velocidade da correnteza $1,6-120,6 \mathrm{~cm} \mathrm{~s}^{-1}(\mathrm{X}=30,4 \pm 29,4) ; \mathrm{pH} \mathrm{6,54-7,44}$ $(\mathrm{X}=7,1 \pm 0,5)$; Oxigênio dissolvido 5,9-6,6 mg L-1 $(\mathrm{X}=6,2 \pm 0,4) ;$ Profundidade 7,0-27,0 cm $(X=15,2-5,5)$.

Material examinado: BRASIL. São PAULo: São João de Iracema, Fazenda São Francisco, 19-VII-2007, F.V.R. Almeida s.n. (SJRP29804).

Esta espécie já foi reportada para o Estado de São Paulo por Sant'Anna \& Azevedo, 1995 (como Microcoleus sociatus), apresentando filamentos bem mais largos (35,0-40,0 ㅆm diâm.). Entretanto, o menor diâmetro do filamento ocorreu essencialmente devido ao tamanho da bainha, o que também foi observado para a espécie Microcoleus subtorulosus, encontrada no mesmo ponto de amostragem. A disposição da bainha pode variar conforme o ambiente, portanto esta característica não comprometeu a identificação da população.

\section{Heterokontophyta}

Vaucheria pseudogeminata Dang., Botaniste 29: 216, 1939.

Figuras 29-31

Talos formando tufos verdes sobre o substrato; sifão 30,0-40,0 $\mu \mathrm{m}$ diâm.; indivíduos monóicos; gametóforo com 2 oogônios laterais a um anterídio central; pedúnculo do gametóforo 100,0-260,0 $\mu \mathrm{m}$ compr., 32,0-44,0 $\mu \mathrm{m}$ diâm.; anterídio cilíndrico-circinado, 40,0-50,0(-60,0) $\mu \mathrm{m}$ compr.,12,0-18,0 $\mu \mathrm{m}$ diâm.; pedicelo do anterídio ereto, levemente curvado; oogônios eretos, imaturos.

Condições ambientais: $(\mathrm{n}=1)$ : Temperatura 19,0-19,4 ${ }^{\circ} \mathrm{C}(\mathrm{X}=19,2 \pm 0,2)$; Condutividade $7,0-8,0 \mu \mathrm{S} \mathrm{cm}^{-1}(\mathrm{X}=7,3 \pm 0,6)$; Turbidez 13,0-30,0 UNT $(\mathrm{X}=18,7 \pm 9,8)$; Velocidade da correnteza $3,0-52,4 \mathrm{~cm} \mathrm{~s}^{-1}(\mathrm{X}=30,0 \pm 13,1) ; \mathrm{pH} \mathrm{6,3-6,5}$ $(\mathrm{X}=6,4 \pm 0,1)$; Oxigênio dissolvido 5,0-6,1 $\mathrm{mg} \mathrm{L}^{-1}$ $(\mathrm{X}=5,4 \pm 0,6)$; Profundidade 2,5-12,0 cm $(\mathrm{X}=8,1 \pm 2,9)$.
Material examinado: BRASIL. São Paulo: Novo Horizonte, Fazenda Serrinha, 14-VII-2007, F.V.R. Almeida s.n. (SJRP29803).

Esta espécie assemelha-se a Vaucheria geminata Vaucher, entretanto a última apresenta dimensões maiores: sifão: (48-)58,0-96,0 $\mu \mathrm{m}$ diâm.; pedúnculo do gametóforo: 172,0-364,0 $\mu \mathrm{m}$ compr., 48,0-84,0 $\mu \mathrm{m}$ diâm.; anterídio: (60,0-)70,0-108,0 $\mu \mathrm{m}$ compr., 24,0-41,0 بm diâm., de acordo com Entwisle (1988). Não foi possível especificar o tamanho dos oogônios e dos oósporos, por encontrarem-se imaturos; nem distinguir a cavidade oogonial, que são características diagnósticas adicionais para a espécie. Entretanto, com base nas demais características, pôde-se identificar a amostra como Vaucheria pseudogeminata. Este é o primeiro registro da espécie para o Brasil, sendo que esta já foi registrada na Austrália (Entwisle 1988), América do Norte (Blum 1972), Europa (Dangeard 1939) e Norte da África (Gauthier-Lievre 1955).

\section{Rhodophyta}

\section{'Chantransia macrospora'}

(Estágio 'Chantransia' de Batrachospermum macrosporum)

Figura 37

Filamentos verde-azulados, com o sistema basal formado por filamentos rizoidais bem desenvolvidos; filamentos compostos por células cilíndricas,

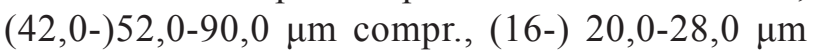
diâm.; ramos formando ângulo fechado com o ramo principal; monosporângios obovóides a esféricos, (18,0-) 26,0-28,0 $\mu \mathrm{m}$ compr., 18,0-26,0 $\mu$ m diâm.

Condições ambientais: $(n=2)$ : Temperatura $16,3-19,8{ }^{\circ} \mathrm{C}(\mathrm{X}=18,9 \pm 1,7)$; Condutividade $30,0-37,0 \mu \mathrm{S} \mathrm{cm}^{-1}(\mathrm{X}=34,2 \pm 3,0)$; Turbidez 9,0-25,0 UNT ( $\mathrm{X}=13,5 \pm 7,7)$; Velocidade da correnteza $0,0-38,5 \mathrm{~cm} \mathrm{~s}^{-1}(\mathrm{X}=6,9 \pm 8,0) ; \mathrm{pH}$ 7,4-7,8 ( $\mathrm{X}=7,7 \pm 0,2)$; Oxigênio dissolvido 4,8-5,2 $\mathrm{mg} \mathrm{L}^{-1}(\mathrm{X}=4,9 \pm 0,2)$; Profundidade $16,0-63,0 \mathrm{~cm}$ $(\mathrm{X}=36,7 \pm 14,5)$.

Material examinado: BRASIL. São PAUlo: Bebedouro, Fazenda Córrego dos Bois, 17-VI-2007, F.V.R. Almeida s.n. (SJRP29707). BRASIL. São PAUlo: Turmalina, Fazenda São João, 17-VIII-2007, F.V.R. Almeida \& O. Necchi Jr. s.n. (SJRP29805).

Conforme recomendação de Zucchi \& Necchi Jr. (2003), associou-se a ocorrência da alga com uma das 


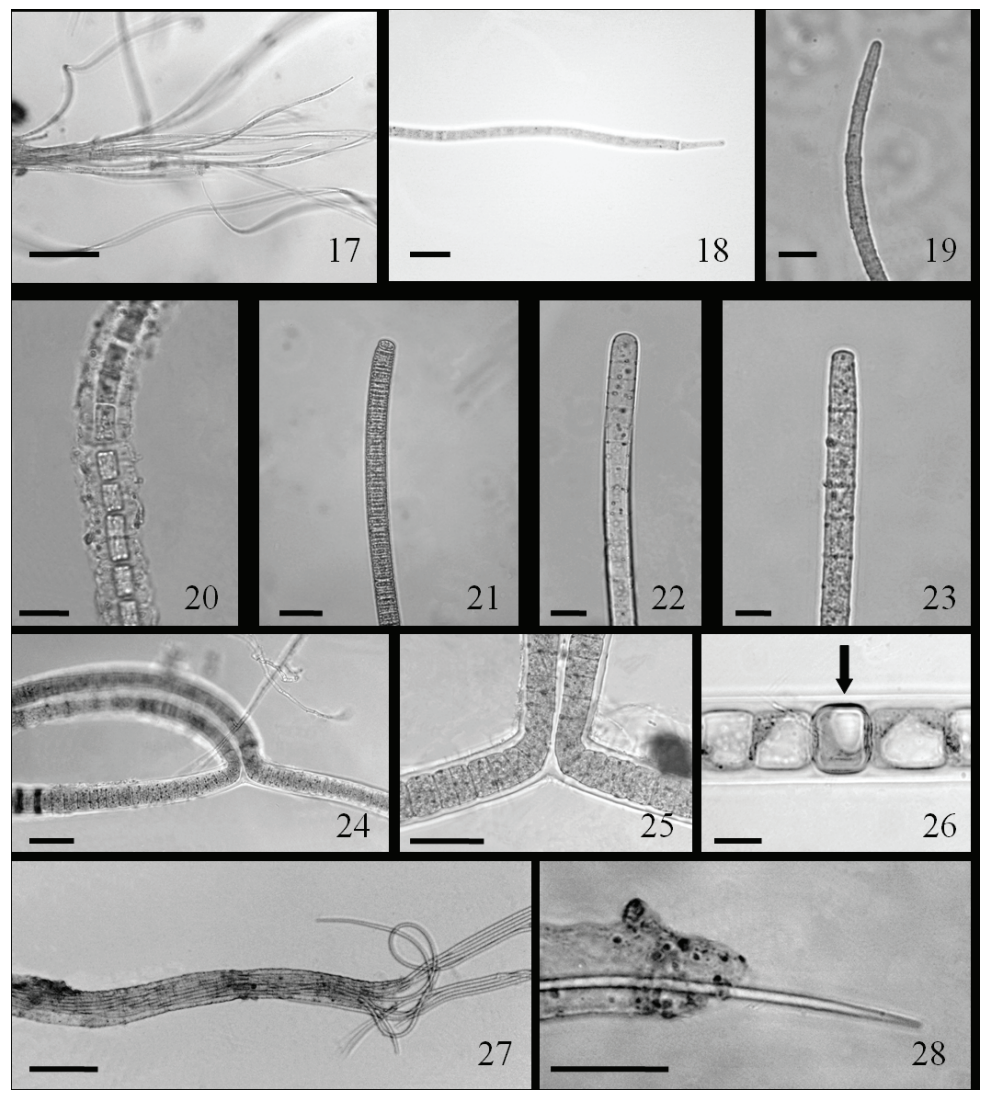

Figuras 17-28. Fotomicrografias das espécies de Cyanophyta descritas. 17-18. Geitlerinema splendidum.17. Aspecto geral de um emaranhado de filamentos. 18. Filamento evidenciando célula apical capitada. 19. Jaaginema homogeneum. 20. Microcoleus subtorulosus. 21. Oscillatoria tenuis. 22. Phormidium retzii. 23. Phormidium cf. retzii. 24-26. Scytonema arcangeli. 24-25. Filamento evidenciando uma ramificação falsa dupla. 26. Detalhe de um heterocito (seta). 27-28. Trichocoleus sociatus. 27. Filamento com muitos tricomas. 28. Detalhe da extremidade de um filamento. (Escalas: Figuras 18-20, 22-23, $26=10 \mu \mathrm{m} ; 21,24-25,28=25 \mu \mathrm{m} ; 17,27=50 \mu \mathrm{m}$ ).

Figures. 17-28. Photomicrographs of Cyanophyta species. 17-18. Geitlerinema splendidum.17. General view of entangled filaments. 18. Filament showing headed appical cell. 19. Jaaginema homogeneum. 20. Microcoleus subtorulosus. 21. Oscillatoria tenuis. 22. Phormidium retzii. 23. Phormidium cf. retzii. 24-26. Scytonema arcangeli. 24-25. Filament showing double false branches. 26. Detail of an heterocyte (arrow). 27-28. Trichocoleus sociatus. 27. Filament with many trichomes. 28. Detail of a filament apex. (Scale bars: Figures $18-20,22-23,26=10 \mu \mathrm{m} ; 21,24-25,28=25 \mu \mathrm{m} ; 17,27=50 \mu \mathrm{m})$.

morfologias referidas para o estágio 'Chantransia', neste caso, 'Chantransia macrospora'. Tal morfologia tem sido relacionada com uma única espécie (B. macrosporum), com base em dados moleculares (Chiasson et al. 2005).

Compsopogon caeruleus (Balbis ex C. Agardh) Montagne, Algues Expl. Sc. Algerie, Bot. 1: 154. 1846.

Figuras 35- 36

Filamentos corticados, com células axiais doliformes ou sub-esféricas; corticação formada verticalmente, por meio de divisões regulares das células axiais, sem filamentos rizoidais; células corticais externas geralmente poligonais; filamentos unisseriados com células terminais discóides;
Condições ambientais: $(\mathrm{n}=1$; $\mathrm{P} 4)$ : Temperatura 19,7-19,8 ${ }^{\circ} \mathrm{C}(\mathrm{X}=19,7 \pm 0,1)$; Condutividade $35,0-37,0 \mu \mathrm{S} \mathrm{cm} \mathrm{cm}^{-1}(\mathrm{X}=35,7 \pm 1,2)$; Turbidez 9,0-10,0 UNT $(X=9,7 \pm 0,6)$; Velocidade da correnteza $0,0-22,4 \mathrm{~cm} \mathrm{~s}^{-1}(X=9,2 \pm 7,9) ; \mathrm{pH}$ 7,7-7,8 $(\mathrm{X}=7,8 \pm 0,1)$; Oxigênio dissolvido $4,8-5,2 \mathrm{mg} \mathrm{L}^{-1}(\mathrm{X}=5,0 \pm 0,2)$; Profundidade $16,0-63,0 \mathrm{~cm}(\mathrm{X}=36,8 \pm 15,0)$.

Material examinado: BRASIL. São PAULO: Turmalina, Fazenda São João, 17-VIII-2007, F.V.R. Almeida \& O. Necchi Jr. s.n. (SJRP29806).

Kumanoa ambigua (Montagne) Entwisle, M.L. Vis, W.B. Chiasson, Necchi Jr. \& A.R. Sherwood, Journal of Phycology 45: 710. 2009.

Figuras 32-34 
Planta monóica, moderadamente mucilaginosa; ramificação irregular, abundante; verticilos doliformes, contíguos, 290-400 $\mu \mathrm{m}$ diâm.; internó com 135-350 $\mu \mathrm{m}$ compr.; ramos primários retos, com 9-11 células; células proximais cilíndricas a elípticas, células distais obovóides a esféricas; ramos secundários numerosos, retos, sobre toda a extensão do internó; espermatângios terminais, esféricos; ramo que sustenta o carpogônio torcido em hélice; tricogínio cilíndrico, pedicelado; carposporófito central, menor que o raio do verticilo.

Condições ambientais: $(\mathrm{n}=1)$ : Temperatura $16,5-16,8{ }^{\circ} \mathrm{C}(\mathrm{X}=16,7 \pm 0,2)$; Condutividade $19,0 \mu \mathrm{S} \mathrm{cm}^{-1}(\mathrm{X}=19,0 \pm 0,0)$; Turbidez 23,0-51,0 UNT $(\mathrm{X}=36,7 \pm 14,0)$; Velocidade da correnteza $0,0-93,8 \mathrm{~cm}$ $\mathrm{s}^{-1}(\mathrm{X}=27,9 \pm 27,8) ; \mathrm{pH} 6,5-6,8(\mathrm{X}=6,7 \pm 0,2)$; Oxigênio dissolvido 6,1-6,4 $\mathrm{mg} \mathrm{L}^{-1}(\mathrm{X}=6,2 \pm 0,2)$; Profundidade $6,0-60,0(X=15,9 \pm 1,6)$.

Material examinado: BRASIL. São PAUlo: Bebedouro, Fazenda Córrego dos Bois, 4-VII-2007, F.V.R. Almeida s.n. (SJRP29706).

Nos 17 riachos amostrados foram encontrados 21 táxons infra-genéricos de macroalgas, incluindo dois grupos vegetativos: Oedogonium spp. (Oedogonium sp.1, Oedogonium sp.2 e Oedogonium sp.3) e Spirogyra spp. (Spirogyra sp.1 e Spirogyra sp.2). Esses táxons pertencem a 14 gêneros e são distribuídas nos filos: Chlorophyta, Cyanophyta, Heterokontophyta e Rhodophyta, sendo estes comumente reportados como típicos de ambientes lóticos (Sheath \& Cole 1992, Necchi Jr. et al. 1997, Krupek et al. 2007, Peres et al. 2008, Necchi Jr. et al. 2008). Chlorophyta (43\%) e Cyanophyta (38\%) e foram os grupos predominantes, o que também tem sido observado em trabalhos anteriores (Sheath \& Burkholder 1985, Sheath et al. 1989, Entwisle 1989, Biggs 1990, Sheath \& Cole 1992, 1996, Necchi Jr. et al. 1997, 2000, 2008, Krupek et al. 2007, Peres et al. 2008). Rhodophyta e Heterokontophyta foram pouco representativos (14 e 5\%, respectivamente), o que ocorreu também em outros estudos realizados no Brasil (Necchi Jr. et al. 1997, Branco \& Necchi Jr. 1998, Necchi Jr. et al. 2000, Krupek et al. 2007, Peres et al. 2008; 8-20\% e 3-11\%, respectivamente).

O número total de espécies do presente estudo (21) foi relativamente baixo, comparado a estudos feitos com metodologia semelhante no noroeste Estado de São Paulo (25-51 espécies; Necchi Jr. et al. 1994b,
Necchi Jr. et al. 1997, 2000). Tal resultado pode ser atribuído ao tipo de substrato, que foi predominantemente arenoso (65\%) e à escassez de luz, pois os riachos encontravam-se em sua maioria em trechos sombreados (53\%). O número de espécies relativo ao número de pontos de amostragem (1,2 neste trabalho) situa-se dentro dos limites dos valores referentes aos trabalhos mencionados $(0,9-1,2)$. Entretanto, há uma tendência de diminuição do número de novas ocorrências com o aumento do número de amostragens, resultante da repetição de espécies (Sheath \& Cole 1992, Branco \& Necchi Jr. 1996, Necchi Jr. et al. 2000).

Os gêneros melhor representados foram Microspora e Nitella, com duas espécies cada. Heterokontophyta foi representado por um único gênero e espécie: Vaucheria pseudogeminata. A maioria das espécies (69\%) foi encontrada exclusivamente em um único ponto de amostragem, e essa alta proporção de espécies exclusivas de um único riacho é um padrão frequentemente observado em comunidades de macroalgas lóticas (Sheath \& Burkholder 1985, Sheath et al. 1986, 1989, Necchi Jr. et al. 1994b, 2000).

Phormidium retzii foi a espécie mais frequente, ocorrendo em seis pontos de amostragem. Esta espécie tem sido reportada como cosmopolita e de ampla distribuição em vários estudos realizados no Brasil (Branco et al. 1999, Necchi Jr. et al. 2000, Krupek et al. 2007), na América do Norte (Sheath \& Cole 1992), Ilhas Fiji (Sheath \& Cole 1996) e no Hawaí (Vis et al. 1994). Algumas espécies encontradas (Kumanoa ambigua, Compsopogon caeruleus e Nitella rosa-mariae) têm sido reportadas como predominantemente tropicais (Necchi Jr. 1991, Necchi Jr. \& Dip 1992, Picelli-Vicentin 2004). Todas as espécies amostradas no presente estudo já foram reportadas para a região noroeste (Necchi Jr. et al. 1991, Necchi Jr. 1993, Necchi Jr. \& Pascoaloto 1993, Necchi Jr. et al. 1994a, b, Necchi Jr. \& Moreira 1995, Branco \& Necchi Jr. 1997, Necchi Jr. et al. 1997, Branco \& Necchi Jr. 1998, Branco et al. 1999, Necchi Jr. et al. 2000, Branco et al. 2001, Branco \& Pereira 2002, Necchi Jr. et al. 2002, Vieira et al. 2002, 2003, Branco et al. 2005), exceto Trichocoleus sociatus, que foi reportada pela primeira vez na região e Vaucheria pseudogeminata (Heterokontophyta), que representa a primeira ocorrência no Brasil.

A proporção dos tipos morfológicos foi: filamentos livres (46\%), emaranhados de filamentos (40\%), filamentos gelatinosos e tufos ( $7 \%$ cada). A predominância de filamentos livres, seguida de 


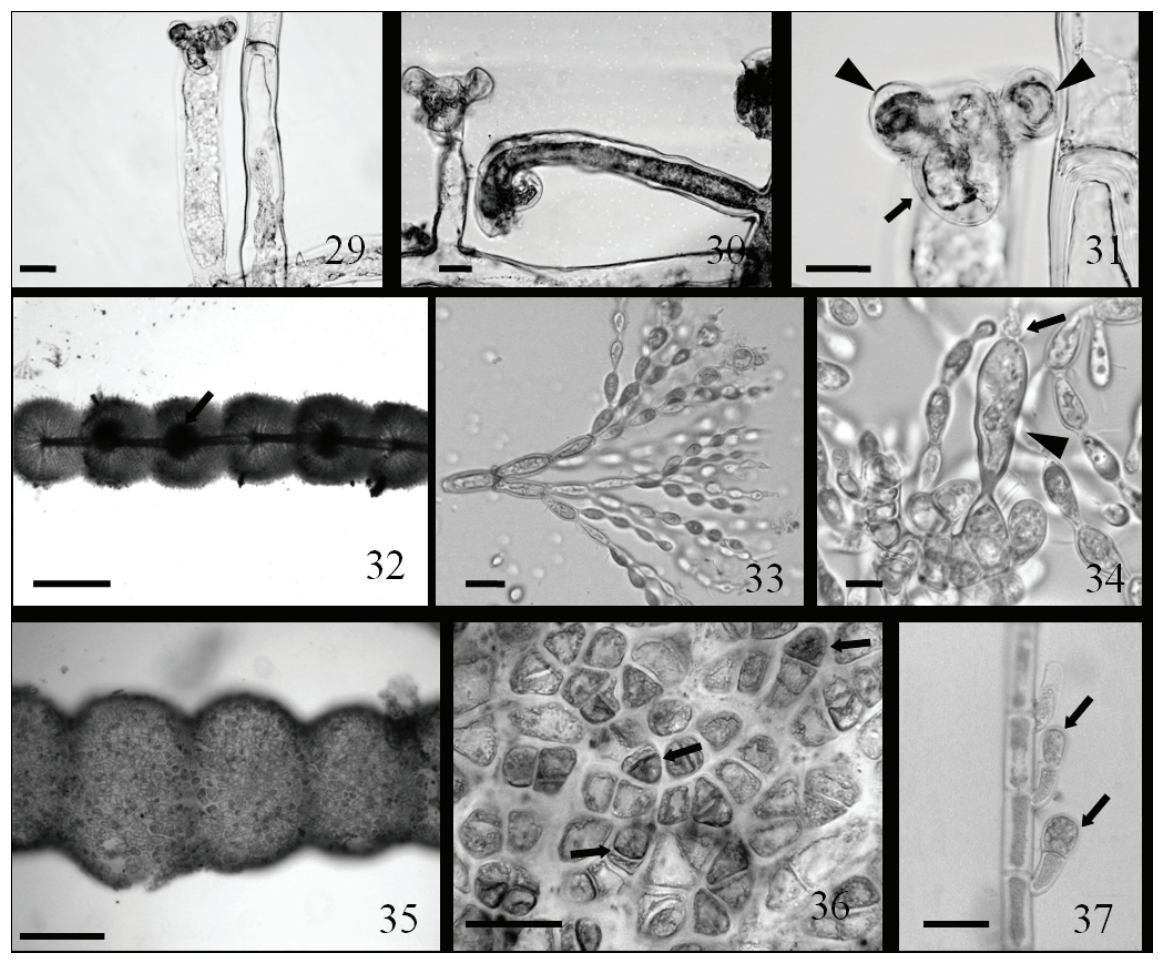

Figuras 29-37. Fotomicrografias das espécies de Heterokontophyta e Rhodophyta descritas. 29-31. Vaucheria pseudogeminata (Heterokontophyta). 29-30. Gametóforos pedunculados. 31. Detalhe do gametóforo: anterídeo circinado (seta), com dois oogônios imaturos laterais (pontas de seta). 32-34. Kumanoa ambigua (Rhodophyta). 32. Aspecto geral dos verticilos, evidenciando carposporófito (seta). 33. Ramo primário. 34. Carpogônio fecundado, evidenciando tricogínio (ponta de seta) com espermácio (seta). 35-36. Compsopogon caeruleus (Rhodophyta). 35. Aspecto geral de um filamento corticado. 36. Detalhe em vista superficial de um filamento corticado evidenciando células corticais e monosporângios (seta). 37. 'Chantransia macrospora' (Rhodophyta). Filamento com monosporângios (setas). (Escalas: Figuras 33-34: $10 \mu \mathrm{m} ; 31,36-37=25 \mu \mathrm{m} ; 29-30=50 \mu \mathrm{m} ; 32,35=200 \mu \mathrm{m}$ ).

Figuras 29-37. Photomicrographs of Heterokontophyta and Rhodophyta species. 29-31. Vaucheria pseudogeminata (Heterokontophyta). 29-30. Stalked gametophores. 31. Detail of a gametophore: circinated antheridium (arrow), with two immature lateral oogonia (arrowheads). 32-34. Kumanoa ambigua (Rhodophyta). 32. General view of whorls, showing carposporophyte (arrow). 33. Primary fascicle. 34. Fertilized carpogonium, showing tricogyne (arrowhead) with espermatium (arrow). 35-36. Compsopogon caeruleus (Rhodophyta). 35. General view of a corticated filament. 36. Detail in a superficial view of a corticated filament showing cortical cells and monosporangia (arrows). 37. 'Chantransia macrospora' (Rhodophyta). Filament with monosporangia (arrows). (Scale bars: Figures 33-34: 10 $\mu \mathrm{m} ; 31$, 36-37 = 25 $\mu \mathrm{m}$; $29-30=50 \mu \mathrm{m} ; 32,35=200 \mu \mathrm{m})$.

emaranhado de filamentos, foi semelhante a estudos realizados no Parque Nacional de Itatiaia, RJ-MG (35 e $24 \%$, respectivamente, Necchi Jr. et al. 2008) e na região noroeste do Estado de São Paulo (40 e 31\%, Necchi Jr. et al. 1997), que corresponde a região estudada no presente trabalho.

Em comparação com estudos realizados em diferentes regiões/biomas do Estado de São Paulo (Necchi Jr. et al. 2000), a flora do presente trabalho assemelhou-se mais àquela de Floresta Tropical, conforme previsto, com nove espécies em comum $(56 \%)$, seguida de Floresta Subtropical, com oito $(50 \%)$, Mata Atlântica, com sete $(44 \%)$ e região de águas duras, com seis espécies em comum (37\%). O bioma que apresentou a menor similaridade de espécies com os fragmentos da região noroeste foi o
Cerrado, com a ocorrência de apenas duas espécies em comum (12\%). Essa baixa similaridade, entretanto, pode ser justificada pelo fato do Cerrado ter sido, entre os biomas amostrados por Necchi Jr. et al. (2000), o que possuiu o menor número de espécies reportadas, reduzindo assim as chances de ocorrência comum de táxons entre os dois biomas.

\section{Agradecimentos}

Aos pesquisadores do laboratório de ficologia da Unesp-São José do Rio Preto, pelos conselhos referentes ao trabalho e auxílio nas coletas; e à FAPESP, pela bolsa concedida à primeira autora do trabalho (Proc. 2007/05919-1) e pelo apoio financeiro para a realização das coletas e equipamentos como parte do projeto temático (Proc. 2004/04820-3) 


\section{Literatura citada}

Biggs, B.J.F. 1990. Periphyton communities and their enviroments in New Zealand rivers. New Zealand Journal of Marine and Freshwater Research 24: 367-386.

Blum, J.L. 1972. Vaucheriaceae. North American Flora (ser.II) 8: 1-64.

Branco, C.C.Z., Branco, L.H.Z., Moura, M.O. \& Bertusso, F.R. 2005. The succession dynamics of a macroalgal community after a flood disturbance in a tropical stream from São Paulo State, southeastern Brazil. Revista Brasileira de Botânica 28: 267-275.

Branco, C.C.Z. \& Necchi Jr., O. 1996. Distribution of stream macroalgae in the eastern Atlantic rainforest of São Paulo State, southeastern Brazil. Hydrobiologia 333: 139-150.

Branco, C.C.Z., Necchi Jr., O. \& Branco, L.H.Z. 2002. Taxonomy and ecological distribution of Chaetophoraceae (Chaetophorales, Chlorophyta) in lotic ecosystems from São Paulo State, southeastern Brazil. Algological Studies 106: 43-75.

Branco, L.H.Z. \& Necchi Jr., O. 1997. Seasonality of macroalgae in three tropical drainage basins in São Paulo State, southeastern Brazil. Archiv für Hydrobiologie 141: 75-91.

Branco, L.H.Z. \& Necchi Jr., O. 1998. Distribution of stream macroalgae in three tropical drainage basins of southeastern Brazil. Archiv für Hydrobiologie 142: 241-256.

Branco, L.H.Z., Necchi Jr., O. \& Branco, C.C.Z. 1999. Cyanophyceae from lotic ecosystems of São Paulo State, southeastern Brazil. Algological Studies 94: 63-87.

Branco, L.H.Z., Necchi Jr., O. \& Branco C.C.Z. 2001. Ecological distribution of Cyanophyceae in lotic ecosystems of São Paulo State. Revista Brasileira de Botânica 24: 99-108.

Branco, L.H.Z. \& Pereira, J.L. 2002. Evaluation of seasonal dynamics and bioindication potential of macroalgal communities in a polluted tropical stream. Archiv für Hydrobiologie 155: 147-161.

Chiasson, W.B., Sabo, N.J. \& Vis, M.L. 2005. Affinities of freshwater putative chantransia stages (Rhodophyta) from molecular and morphological data. Phycologia 44: 163-168.

Dangeard, P. 1939. Le genre Vaucheria, spécialement dans la région du sud-ouest de la France. Botaniste 29: 183-265.

Entwisle, T.J. 1988. A monograph of Vaucheria (Vaucheriaceae, Chrysophyta) in south-eastern Mainland Australia. Australian Systematic Botany 1: 1-77.

Entwisle, T.J. 1989. Macroalgae in Yarra River basin: flora and distribution. Proceedings of the Royal Society of Victoria 101: 1-76.
Gauthier-Lievre, L. 1955. Le genre Vaucheria en Afrique du Nord. Bulletin de la Société d'Histoire Naturelle de l'Afrique du Nord 46: 301-31.

Geitler, L. 1932. Cyanophyceae. In: T.L. Rabenhorst (ed.). Kryptogamenflora von Deutschland, Österreich und der Schweiz. Akademishe Verlagsgesellschaft, Leipzig 14: 1-1196.

Graham, L.E., Graham, J.M. \& Wilcox, L.W. 2009. Algae. 2 ed. Pearson Education, San Francisco.

Holmgren, P.K. \& Holmgren, N.H. 2001. Index Herbariorum: a global directory of public herbaria and associated staff. New York Botanical Garden's Virtual Herbarium. http://sciweb.nybg.org/science2/ IndexHerbariorum.asp (acesso em 09.05.2011).

Holmgren, P.K. \& Holmgren, N.H. 1993. Additions to Index Herbariorum (Herbaria), ed. 8, second series. Taxon 42: 489-505.

Krupek, R.A., Branco, C.C.Z. \& Peres, C.K. 2007. Distribuição ecológica das comunidades de macroalgas da bacia de drenagem do rio das Pedras, região centro-sul do Estado do Paraná, Sul do Brasil. Revista Brasileira de Botânica 30: 173-182.

Laurence, W.F. \& Yensen, E. 1991. Predicting the impacts of edge effects in fragmented habitats. Biological Conservation 55: 45-67.

Necchi Jr., O. 1991. The section Sirodotia of Batrachospermum (Rhodophyta, Batrachospermaceae) in Brazil. Algological Studies 62: 17-30.

Necchi Jr., O. 1993. Distribution and seasonal dynamics of Rhodophyta in the Preto River basin, southeastern Brazil. Hydrobiologia 250: 81-90.

Necchi Jr., O. 2004. Amostragem de algas bentônicas. In: C.E.M. Bicudo \& D.C. Bicudo (Eds.). Amostragem em limnologia. RIMA Editora, São Carlos, pp.167-177.

Necchi Jr., O., Branco, C.C.Z. \& Branco, L.H.Z. 1999. Distribution of Rhodophyta in streams from São Paulo State, southeastern Brazil. Archiv für Hydrobiologie 147: 73-89.

Necchi Jr., O., Branco, C.C.Z. \& Branco, L.H.Z. 2000. Distribution of stream macroalgae in São Paulo State, Southeastern Brazil. Algological Studies 97: 43-57.

Necchi Jr., O., Branco, C.C.Z., Simão, R.C.G. \& Branco, L.H.Z. 1995. Distribution of stream macroalgae in the northwest region of São Paulo State, southeastern Brazil. Hydrobiologia 299: 219-230.

Necchi Jr., O., Branco, L.H.Z. \& Branco, C.C.Z. 2003. Ecological distribution of stream macroalgal communities from a drainage basin in the Serra da Canastra National Park, Minas Gerais, southeastern Brazil. Brazilian Journal of Biology 63: 635-646.

Necchi Jr., O., Branco, L.H.Z. \& Dip, M.R. 1994a. Uso de macroalgas para avaliação da poluição orgânica no Rio Preto, noroeste do Estado de São Paulo. Anais da Academia Brasileira de Ciências 66: 359-371. 
Necchi Jr., O., Branco, L.H.Z. \& Pascoaloto, D. 1994b. Distribution of macroalgae in a tropical river basin from southeastern Brazil. Archiv für Hydrobiologie 129: 459-471.

Necchi Jr., O., Branco, L.H.Z. \& Spezamiglio, D.N. 2008. Ecological distribution of stream macroalgal communities from "Parque Nacional de Itatiaia", states of Minas Gerais and Rio de Janeiro, Brazil. Revista Brasileira de Botânica 31: 135-145.

Necchi Jr., O. \& Dip, M.R. 1992. The family Compsopogonaceae (Rhodophyta) in Brazil. Algological Studies 66: 105-118.

Necchi Jr., O., Dip, M.R. \& Goes, R.M. 1991. Macroalgae of a stream in southeastern Brazil: composition, seasonal variation and relation to physical and chemical variables. Hydrobiologia 213: 241-250.

Necchi Jr., O. \& Moreira, J.C.L.1995. Longitudinal distribution of macroalgae in two tropical lotic ecosystems from southeastern Brazil. Archiv für Hydrobiologie 135: 113-128.

Necchi Jr., O. \& Pascoaloto, D. 1993. Seasonal dynamics of macroalgal communities in the Preto River basin, São Paulo, southeastern Brazil. Archiv für Hydrobiologie 129: 231-252.

Necchi Jr., O., Pascoaloto, D., Branco, C.C.Z. \& Branco, L.H.Z. 1997. Stream macroalgal flora from the northwest region of São Paulo State, Southeastern Brazil. Algological Studies 84: 91-112.

Necchi Jr., O., Spezamiglio, D.N., Branco, C.C.Z. \& Branco, L.H.Z. 2001. Estudo taxonômico e distribuição ecológica do gênero Vaucheria (Xanthophyta, Vaucheriaceae) em ecossistemas lóticos do Estado de São Paulo, Brasil. Hoehnea 28: 231-242.

Necchi Jr., O., Spezamiglio, D.N., Branco, C.C.Z. \& Branco, L.H.Z. 2002. Taxonomy and ecological distribution of the genus Microspora (Microsporaceae, Chlorophyta) in lotic ecosystems of São Paulo State, Southeastern Brazil. Algological Studies 105: 39-50

Peres, C.K., Branco, C.C.Z. \& Krupek, R.A. 2008. Macroalgas de riachos da Serra da Prata, leste do Estado do Paraná, Sul do Brasil. Acta Botanica Brasilica 22: 333-344.

Picelli-Vicentim, M.M., Bicudo, C.E.M. \& Bueno, N.C. 2004. Flora ficológica do Estado de São Paulo 5: Charophyceae. RIMA Editora, São Carlos.

Probio. 1998. Áreas de domínio do cerrado no Estado de São Paulo. Imprensa Oficial, Secretaria do Meio Ambiente de São Paulo, São Paulo.
Rambaldi, D.M. \& Oliveira, D.A.S. 2003. Fragmentação de ecossistemas: causas, efeitos sobre a biodiversidade e recomendações de políticas públicas. Ministério do Meio Ambiente, Brasília.

Round, F.E. 1965. The biology of algae. Edward Arnold, London.

Sant'Anna, C.L.\& Azevedo, M.T.P. 1995. Oscillatoraceae (Cyanophyceae) from São Paulo State, Brazil. Nova Hedwigia 60: 18-58.

Sheath, R.G. \& Burkholder, J. 1985. Characteristics of softwater streams in Rhode Island. II: Composition and seasonal dynamics of macroalgae communities. Hydrobiologia 128: 109-118.

Sheath, R.G. \& Cole, K.M. 1992. Biogeography of stream macroalgae in North American. Journal of Phycology 28: 448-460.

Sheath, R.G. \& Cole, K.M. 1996. Stream macroalgae of the Fiji Islands: A preliminary study. Pacific Science 50: 46-54.

Sheath, R.G., Hamilton, P.B., Hambrook, J.A. \& Cole, K.M. 1989. Stream macroalgae of the eastern boreal forest region of North America. Canadian Journal of Botany 67: 3353-3362.

Sheath, R.G., Morinson, M.O., Korsh, J.E., Kaczmarczyk, D. \& Cole, K.M. 1986. Distribution of stream macroalgae in south-central Alaska. Hydrobiologia 135: 259-269.

Strahler, A.N. 1957. Quantitative analysis of watershed geomorphology. American Geophysical Union Transation 38: 913-920.

Vieira, J.Jr., Necchi Jr., O., Branco, C.C.Z. \& Branco, L.H.Z. 2002. Characeae (Chlorophyta) em ecossistemas lóticos do Estado de São Paulo, Brasil: gênero Nitella. Hoehnea 29: 249-266.

Vieira, J.Jr., Necchi Jr., O., Branco, C.C.Z. \& Branco, L.H.Z. 2003. Characeae (Chlorophyta) em ecossistemas lóticos do Estado de São Paulo, Brasil: gênero Chara e distribuição ecológica. Hoehnea 30: 53-70.

Vis, M.L., Sheath, R.G., Hambrook, J.A. \& Cole, K.M. 1994. Stream macroalgae of the Hawaiian islands: a preliminary study. Pacific Science 48: 175-18.

Zucchi M.R. \& Necchi Jr., O. 2003. Blue-greenish acrochaetioid algae in freshwater habitats are 'Chantransia' stages of Batrachospermales sensu lato (Rhodophyta). Cryptogamie, Algologie 24: 117-131. 